$4-1-2011$

\title{
Radial Motion in A Central Potential for Singular Mass Densities
}

Ulrich Zürcher

Cleveland State University, u.zurcher@csuohio.edu

Miron Kaufman

Cleveland State University, m.kaufman@csuohio.edu

Follow this and additional works at: https://engagedscholarship.csuohio.edu/sciphysics_facpub

Part of the Physics Commons

How does access to this work benefit you? Let us know!

Publisher's Statement

(C) 2011 American Association of Physics Teachers

\section{Repository Citation}

Zürcher, Ulrich and Kaufman, Miron, "Radial Motion in A Central Potential for Singular Mass Densities" (2011). Physics Faculty Publications. 277.

https://engagedscholarship.csuohio.edu/sciphysics_facpub/277

This Article is brought to you for free and open access by the Physics Department at EngagedScholarship@CSU. It has been accepted for inclusion in Physics Faculty Publications by an authorized administrator of

EngagedScholarship@CSU. For more information, please contact library.es@csuohio.edu. 


\title{
Radial motion in a central potential for singular mass densities
}

\author{
Ulrich Zürcher and Miron Kaufman
}

\section{INTRODUCTION}

The motion in the gravitational field of a sphere with uniform and constant mass density is discussed in introductory and intermediate-level mechanics texts. ${ }^{1}$ The mass inside the radius $r$ increases as $M(r) \sim r^{3}$, the corresponding gravitational potential is $V(r) \sim r^{2}$, and the radial motion is harmonic. $^{2}$ A sphere with uniform mass density and a point mass (the Kepler problem) are the two gravitational cases that lead to closed orbits, that is, the periods for orbital and radial motion are degenerate.

The radial motion applies to the fictional case when a person falls through a hole through the center of the Earth, popularized in Carroll's Alice in Wonderland ${ }^{3}$ and Baum's Dorothy and the Wizard of $\mathrm{Oz}^{4}{ }^{4}$ The fall through a tunnel goes back to Bacon and Voltaire ${ }^{5}$ and was discussed by Galilei. ${ }^{6}$ The Earth has a shell structure with a mass density increasing toward the center, ${ }^{2}$ and hence the motion is not strictly harmonic in this fictional case.

In this paper, we consider central potentials of the form $M(r) \sim r^{\alpha}$ with $0<\alpha<3$. A point mass corresponds to $M(r) \sim r^{0}$. The mass distribution $M(r)$ defines a local density $\rho(r)$ so that $d M=4 \pi r^{2} \rho(r) d r$. Thus, a power law $M(r) \sim r^{\alpha}$ with $\alpha<3$ corresponds to a density that diverges near the center, $\rho(r) \rightarrow \infty$ for $r \rightarrow 0$. The cases $M(r) \sim r$ and $M(r)$ $\sim r^{2}$ are used to describe the mass distributions in elliptical galaxies. ${ }^{7}$ The determination of mass distributions from measured luminosities goes back to the work by Shapley and de Vaucouleurs. ${ }^{8,9}$ They determined the mass distribution of elliptical galaxies based on the observed luminosities and proposed the form $\rho(r) \sim r^{-3 / 2}$ near the center of the galaxies. It was later found that $\rho(r) \sim r^{-1}$ applies to M32, the small companion of the Andromeda galaxy. Gigantic galaxies, such as M87 in the Virgo cluster, are described by $\rho(r) \sim r^{-2} \cdot{ }^{10} \mathrm{~A}$ singular mass density is described by Dehnen's law, ${ }^{11}$

$$
\rho(\widetilde{r}) \sim \widetilde{r}^{\gamma}(1+\widetilde{r})^{-(4-\gamma)},
$$

where $\widetilde{r}^{2}=x^{2} / a^{2}+y^{2} / b^{2}+z^{2} / c^{2}$. Equation (1) is known as Hernquist's law ${ }^{12}$ for $\gamma=1$ and Jaffe's law for $\gamma=2 .{ }^{13}$ Dehnen's law describes the mass density of the stars and interstellar gas in the elliptical galaxy. We note that mass density has the asymptotic behavior $\rho(\widetilde{r}) \sim \widetilde{r}^{-4}$ for $\tilde{r} \gg 1$. Because black holes do not contribute to the observed luminosity, the density $\rho(\widetilde{r})$ does not describe the (supermassive) black holes that are believed to be at the center of elliptical galaxies.

Although we will not address any specific astrophysical problem, we adopt astrophysical terminology throughout this paper and use the terms "weak" and "strong cusp" for $\gamma$ $=1,2$, respectively. The reader is referred to Ref. 7 for further explanation of the terminology.

\section{GRAVITATIONAL POTENTIAL}

In the following, we use $\mathbf{r}$ for the radial coordinate along the diameter so that $|\mathbf{r}|=r$ is the radial coordinate $r$. The acceleration $a(r)$ of a star at the radius $r$ can be expressed in terms of the mass inside $r$ given by $M(r)=4 \pi \int_{0}^{r} s^{2} \rho(s) d s,{ }^{2}$

$$
\mathbf{a}(\mathbf{r})=-\frac{G M(r)}{r^{2}} \hat{\mathbf{r}},
$$

where $G$ is the gravitational constant and $\hat{\mathbf{r}}$ is a unit vector. The potential $V$ is given by $a(r)=-d V / d r$. We note that $V(r) \neq-G M(r) / r$ if $d M / d r \neq 0$. For a uniform sphere of radius $R$, we have $\rho(r)=\rho_{0}$ for $r<R$ and $\rho(r)=0$ for $r>R$. The potential is given $\mathrm{by}^{2}$

$$
V(r)= \begin{cases}-\left(G \tilde{M} / 2 R^{3}\right)\left(3 R^{2}-r^{2}\right) & (r<R) \\ -G \tilde{M} / r & (r>R),\end{cases}
$$

where the total mass $\tilde{M}$ is $\tilde{M}=M(R)$. The constant $3 G \tilde{M} / R$ is chosen so that the potential is continuous at $r=R$.

We consider the case when the object moves along the diameter so that when the object is released from rest at $r_{0}$ $<R$, the motion is restricted to $-r_{0}<\mathbf{r}(t)<r_{0}$. Because $V(r)$ in Eq. (3) is quadratic, the object undergoes harmonic motion, $\ddot{\mathbf{r}}=-\left(G \tilde{M} / R^{3}\right) \mathbf{r}$. The period is given by

$$
T=2 \pi \sqrt{\frac{R^{3}}{G \tilde{M}}} .
$$

That is, $T^{2} / R^{3}=4 \pi^{2} G \tilde{M}$, which is Kepler's third law.

Although the mass density can be singular at the origin, $\rho(r) \sim r^{-\gamma}$ for $r \rightarrow 0$, the mass inside $r$ must remain finite, that is, $M(r)<\infty$. It follows that $\gamma<3$ in Eq. (1). We introduce a radial scale $R_{0}$ for the singular behavior, $\rho(r) / \rho_{0} \sim\left(R_{0} / r\right)^{\gamma}$, and a radial cutoff $R_{1}$ such that the density follows the behavior for large radii, $\rho(r) / \rho_{0} \sim R_{0}^{\gamma} R_{1}^{4-\gamma} / r^{4}$ for $r>R_{1}$. We will see in the following that $\rho_{0}$ depends on the total mass of the galaxy and the two cutoff radii, $\rho_{0}=M_{\text {tot }} / R_{0}^{\gamma} R_{1}^{3-\gamma}$, up to a constant of order of unity. In the following, we use Dehnen's law in the form,

$$
\rho(r)=\rho_{0}\left(\frac{R_{0}}{r}\right)^{\gamma} \frac{1}{\left(1+r / R_{1}\right)^{4-\gamma}} \quad(\gamma<3) .
$$

The density $\rho(r)$ diverges as $r \rightarrow 0$ and $\rho \rightarrow 0$ as $r \rightarrow \infty$. 
The power-law behavior $\left(R_{0} / r\right)^{\gamma}$ is scale-invariant, and the radius $R_{1}$ is the only characteristic length for the mass distribution of the elliptical galaxy. We have

$$
M(r)=4 \pi \rho_{0} R_{0}^{\gamma} R_{1}^{3-\gamma} \mu\left(r / R_{1}\right),
$$

where we have introduced the function $\mu(z)$ with the dimensionless radius $z=r / R_{1}$,

$$
\mu(z)=\int_{0}^{z} \frac{s^{2-\gamma}}{(1+s)^{4-\gamma}} d s .
$$

For $\gamma=1,2$, we find

$$
M(r)=\tilde{M}\left\{\begin{array}{cc}
\left(r / R_{1}\right)^{2} /\left(1+r / R_{1}\right)^{2} & (\gamma=1) \\
\left(r / R_{1}\right) /\left(1+r / R_{1}\right) & (\gamma=2),
\end{array}\right.
$$

where $\tilde{M}=2^{\gamma} \pi \rho_{0} R_{0}^{\gamma} R_{1}^{3-\gamma}$. The mass $\tilde{M}$ and the radius $R_{1}$ define the characteristic time scale analogous to Eq. (4),

$$
\tilde{T}=\sqrt{\frac{R_{1}^{3}}{G \tilde{M}}} .
$$

The magnitude of the acceleration of an object at the radius $r$ is

$$
a(r)=\widetilde{a} \begin{cases}1 /\left[1+r / R_{1}\right]^{2} & (\gamma=1) \\ 1 /\left[\left(r / R_{1}\right)\left(1+r / R_{1}\right)\right] & (\gamma=2) .\end{cases}
$$

The characteristic scale for acceleration is given by

$$
\tilde{a}=\frac{G \tilde{M}}{R_{1}^{2}} .
$$

The acceleration at the origin is finite for $\gamma=1, a(r \rightarrow 0)$ $\rightarrow \tilde{a}$, and diverges for $\gamma=2, a(r \rightarrow 0)=\widetilde{a} R_{1} / r \rightarrow \infty$.

The potential $V(r)$ follows from Eq. (3),

$$
V(r)=-\widetilde{v}^{2} \begin{cases}1 /\left(1+r / R_{1}\right) & (\gamma=1) \\ \ln \left(1+R_{1} / r\right) & (\gamma=2),\end{cases}
$$

where $\tilde{v}$ is the characteristic speed,

$$
\tilde{v}=\frac{R_{1}}{\tilde{T}}=\sqrt{\frac{G \tilde{M}}{R_{1}}} .
$$

The radial dependence of the potential is different for $r$ $>R_{1}$ and $r<R_{1}$. For $r / R_{1}>1$, we have $\ln \left(1+R_{1} / r\right) \simeq R_{1} / r$ so that

$$
V(r)=-\frac{G \tilde{M}}{r} \quad\left(r \gg R_{1}\right) .
$$

We conclude that the motion of a star far from the center of the elliptical galaxy reduces to the Kepler problem. Therefore, we limit our subsequent discussion to the case when the star is deep inside the galaxy and "feels" the singular mass distribution. We choose the initial condition $r_{0} / R_{1}=0.2$ so that $M\left(r_{0}\right)=\tilde{M} / 36$ and $M\left(r_{0}\right)=\tilde{M} / 6$ in the weak- and strongcusp limits, respectively.

We plot the scaled potential $V(r) / \widetilde{v}^{2}$ for $0<r / R_{1}<0.2$ in Fig. 1. The potential is nearly constant for $\gamma=1$ so that the speed of the star remains finite at all times. For $\gamma=2$, the potential diverges logarithmically so that the speed diverges as the star approaches the center of the galaxy. This divergence is unphysical because the description of the stars' dy-

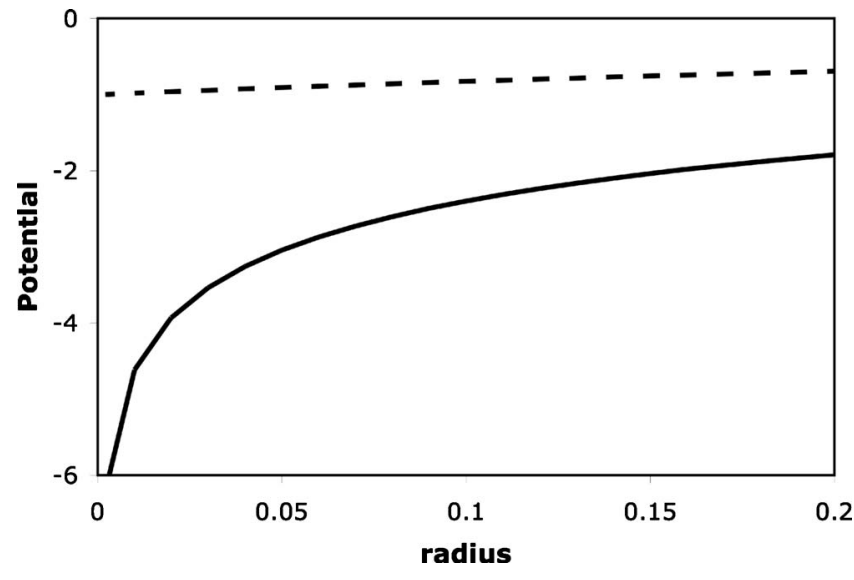

Fig. 1. The gravitational potential $V(r)$ for $\gamma=1$ (dashed) and $\gamma=2$ (solid) from Eq. (15).

namics using Newtonian mechanics must be replaced by a formulation using general relativity or the singular mass density must be regularized.

\section{RADIAL MOTION}

Because the energy is conserved, the solution can be found by integration. Conservation of energy yields the speed $v$ $=|\mathbf{v}|$ for $\gamma=1$,

$$
-\widetilde{v}^{2} \frac{1}{1+r_{0} / R_{1}}=-\widetilde{v}^{2} \frac{1}{1+r / R_{1}}+\frac{1}{2} v^{2}
$$

so that

$$
\frac{v^{2}}{2 \widetilde{v}^{2}}=\frac{r_{0} / R_{1}-r / R_{1}}{\left(1+r_{0} / R_{1}\right)\left(1+r / R_{1}\right)} .
$$

We set $r=0$ and find the maximum speed of the star as it passes through the center of the galaxy,

$$
\frac{v_{\max }}{\tilde{v}}=\sqrt{\frac{2 r_{0} / R_{1}}{1+r_{0} / R_{1}}}
$$

so that $v_{\max } / \widetilde{v} \simeq 0.58$ for $r_{0} / R_{1}=0.2$. Because $x /(1+x)<1$ for $x>0$, we find

$$
v_{\max }<\sqrt{2} \tilde{v}
$$

for arbitrary values of the ratio $r_{0} / R_{1}$.

The equation of motion can be integrated to find the time $t=\int d r / v$ or

$$
\frac{t}{\tilde{T}}=\frac{1}{\sqrt{2}} \sqrt{\frac{R_{1}+r_{0}}{R_{1}}} \int_{r / R_{1}}^{r_{0} / R_{1}} \sqrt{\frac{1+s}{\left(r_{0} / R_{1}\right)-s}} d s .
$$

The remaining integral is elementary,

$$
\begin{aligned}
\frac{t}{\tilde{T}}= & \frac{1}{\sqrt{2}}\left(\frac{R_{1}+r_{0}}{R_{1}}\right)^{3 / 2}\left[\frac{\sqrt{\left(R_{1}+r\right)\left(r_{0}-r\right)}}{R_{1}+r_{0}}\right. \\
& \left.+\sin ^{-1} \sqrt{\frac{r_{0}-r}{R_{1}+r_{0}}}\right] .
\end{aligned}
$$

Equation (20) can be inverted numerically to find the radius as a function of time, $r=r(t)$. We set $r=0$ and find for the period 


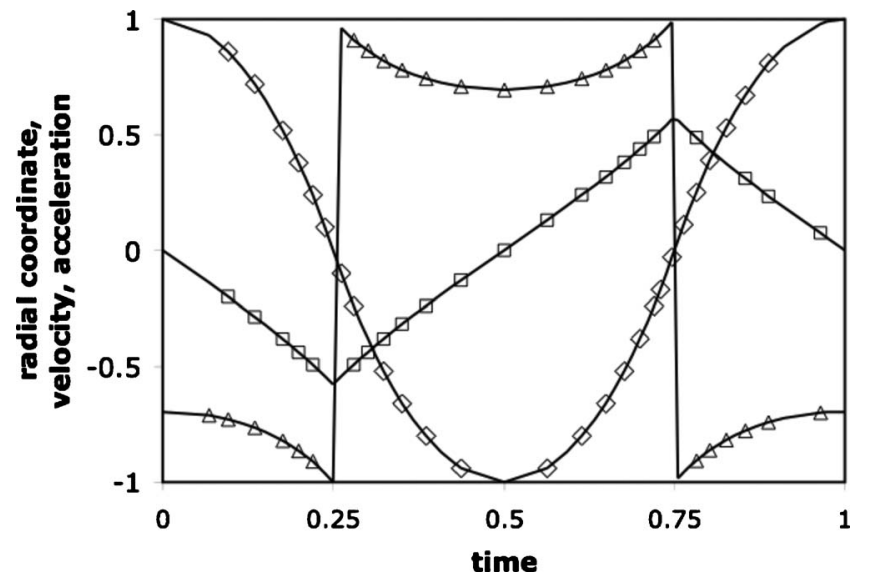

Fig. 2. The scaled radial coordinate $(\diamond)$, velocity $(\square)$, and acceleration $(\triangle)$ in the weak-cusp limit $\gamma=1$.

$$
\frac{T}{\widetilde{T}}=2 \sqrt{2}\left(\frac{R_{1}+r_{0}}{R_{1}}\right)^{3 / 2}\left[\frac{\sqrt{R_{1} r_{0}}}{R_{1}+r_{0}}+\sin ^{-1} \sqrt{\frac{r_{0}}{R_{1}+r_{0}}}\right] .
$$

For small amplitudes, $r_{0} / R_{1}<1$, and we have in leading order,

$$
\frac{T}{\widetilde{T}} \simeq 4 \sqrt{2} \sqrt{\frac{r_{0}}{R_{1}}} .
$$

We now consider the case when the star goes through the center of the galaxy, $t \rightarrow T / 4$. We introduce the small time difference $\Delta t$ as

$$
\Delta t=\frac{T}{4}-t>0
$$

We find from Eq. (20) that

$$
r=\tilde{v} \sqrt{\frac{2 r_{0} / R_{1}}{1+r_{0} / R_{1}}} \Delta t=v_{\max } \Delta t
$$

where we have substituted the expression for the maximum speed of the star [Eq. (17)].

We use MATHCAD (Ref. 14) to compute the timedependent radius, velocity, and acceleration of the star. In Fig. 2, we plot $\mathbf{r} / r_{0}, \mathbf{v} / \tilde{v}$, and $\mathbf{a} / \tilde{a}$ versus $t / T$. Although the time-dependent radius approximately corresponds to harmonic motion, the velocity and acceleration do not; the velocity is similar to a sawtooth function rather than a sine function, and the acceleration is approximately piecewise constant.

We plot the phase portrait of the motion in Fig. 3. The phase diagram is roughly similar to the elliptical shape of the phase portrait for a harmonic oscillator. We observe a cusp when the star passes through the center $\left(r=0, v_{\max }\right)$, which reflects the discontinuity of the star's acceleration, $\mathbf{a}(0+\delta r)$ $=-\mathbf{a}(0-\delta r)=-\tilde{a}$ for $\delta r \rightarrow 0$. There is no cusplike behavior for the harmonic oscillator because the acceleration is zero at $r=0$.

We now consider $\gamma=2$. We have for the conservation of energy,

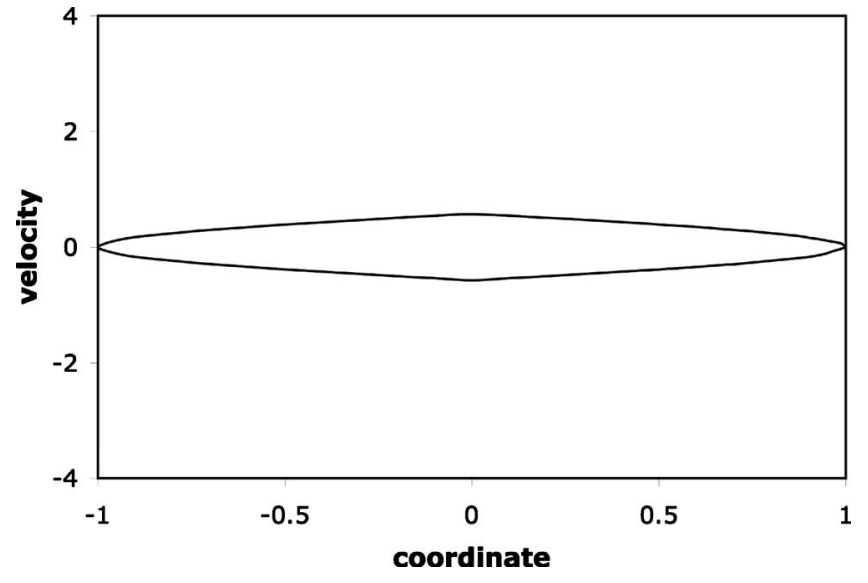

Fig. 3. The phase portrait for the weak-cusp limit.

$$
-\widetilde{v}^{2} \ln \left(1+\frac{R_{1}}{r_{0}}\right)=-\widetilde{v}^{2} \ln \left(1+\frac{R_{1}}{r}\right)+\frac{1}{2} v^{2} .
$$

We find for the speed $v$,

$$
v=\sqrt{2} \widetilde{v} \sqrt{u\left(r / R_{1}\right)-u\left(r_{0} / R_{1}\right)},
$$

where we have introduced the function $u(x)$ for $x>0$,

$$
u(x)=\ln \left(1+\frac{1}{x}\right)>0 .
$$

We write $u_{0}=u\left(r_{0} / R_{1}\right)>1$. Note that $u \simeq \ln \left(R_{1} / r\right) \rightarrow \infty$ for $r \rightarrow 0$. It follows that the speed diverges as the star approaches the center of the elliptical galaxy. This behavior is unphysical because the speed cannot exceed the speed of light. The Schwarzschild radius is obtained by setting $c^{2} / 2$ $=G \tilde{M} / \mathcal{R}_{s}{ }^{15}$

$$
\mathcal{R}_{s}=\frac{2 G \tilde{M}}{c^{2}}
$$

An object smaller than its Schwarzschild radius collapses and becomes a black hole. We set $v=c$ in Eq. (25) and find the radius $r_{c}$,

$$
r_{c}=R_{1} \exp \left(-\frac{R_{1}}{\mathcal{R}_{s}}\right) .
$$

The Schwarzschild radius is the smallest length scale in the problem so that $R_{1} \gg \mathcal{R}_{s}$. It follows that the ratio $r_{c} / R_{1}$ is exponentially small.

Because $r<r_{0}$, we have $u\left(r / R_{1}\right)>u_{0}$, and the time $t$ can be written as

$$
\frac{t}{\tilde{T}}=\frac{1}{\sqrt{2}} \int_{r / R_{1}}^{r_{0} / R_{1}} \frac{1}{\sqrt{u(s)-u_{0}}} d s .
$$

We use $u(x) \simeq-\ln (x)$ for small arguments and find

$$
\frac{t}{\tilde{T}}=2^{-1 / 2} \int_{0}^{\sqrt{u-u_{0}}}\left[\cosh \left(w^{2}+u_{0}\right)-1\right]^{-1} d w .
$$

We use a series expansion for the hyperbolic cosine function and retain the first two terms, $\left[\cosh \left(w^{2}+u_{0}\right)-1\right]^{-1}$ $\simeq 2 \exp \left(-w^{2}-u_{0}\right)+4 \exp \left(-2 w^{2}-2 u_{0}\right)$. The remaining integrals yield error functions, ${ }^{16}$ 


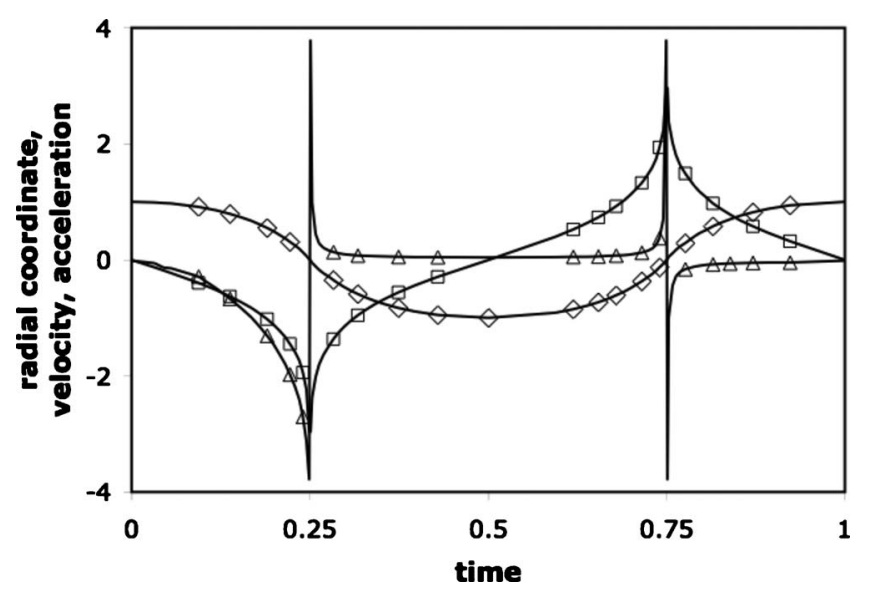

Fig. 4. The scaled radial coordinate $(\diamond)$, velocity $(\square)$, and acceleration $(\triangle)$ in the strong-cusp limit $\gamma=2$.

$$
\frac{t}{\tilde{T}}=\sqrt{\frac{\pi}{2}}\left[e^{-u_{0}} \operatorname{erf}\left(\sqrt{u-u_{0}}\right)+2 e^{-2 u_{0}} \operatorname{erf}\left(\sqrt{2\left[u-u_{0}\right]}\right)\right]
$$

The period follows in the limit $u \rightarrow \infty, \quad T / \widetilde{T}=4 \sqrt{\pi / 2}$ $\left[\exp \left(-u_{0}\right)+2 \exp \left(-2 u_{0}\right)\right]$. Because $\exp \left(-u_{0}\right)=1 /\left(1+R_{1} / r_{0}\right)$, we find $T / \widetilde{T}=2 \sqrt{2 \pi}\left(3+R_{1} / r_{0}\right) /\left(1+R_{1} / r_{0}\right)^{2}$, or in leading order of $r_{0} / R_{1}$,

$$
\frac{T}{\widetilde{T}} \simeq 2 \sqrt{2 \pi} \frac{r_{0}}{R_{1}}
$$

We now consider the case where the star passes through the center of the galaxy, that is, $t \rightarrow T / 4$, and write and define $\Delta t$ as in Eq. (23). We use the asymptotic approximation for the error function, ${ }^{16} \operatorname{erfc}(z)=\pi^{-1 / 2} z^{-1} \exp \left(-z^{2}\right)$, and find in leading order

$$
\tilde{v} \Delta t=\frac{1}{\sqrt{2} \ln ^{1 / 2}\left(r_{0} / r\right)} r
$$

or, alternatively, $r=v_{\text {eff }} \Delta t$ with the $r$-dependent speed, $v_{\text {eff }}$ $=\sqrt{2} \tilde{v} \ln ^{1 / 2}\left(r_{0} / r\right)$. We set $r$ equal to the cutoff radius in Eq. (29) and find the corresponding cutoff time, $\Delta t_{\mathrm{c}} / \tilde{T}$ $=\frac{1}{2} \sqrt{R_{1} / \mathcal{R}_{s}} \exp \left(-R_{1} / \mathcal{R}_{s}\right)$. It follows that $\Delta t_{\mathrm{c}} / \tilde{T} \rightarrow 0$ for $R_{1} / \mathcal{R}_{s} \gg 1$. Equation (26) gives the velocity as a function of $r, v^{2} / 2 \widetilde{v}^{2} \simeq \ln \left(r_{0} / r\right)$. If we substitute Eq. (26) into Eq. (34), we obtain $\Delta t / \tilde{T}=(\tilde{v} / v) \exp \left(-v^{2} / 2 \widetilde{v}^{2}\right)$, indicating that the rapid increase in the star's speed occurs over a very short time as it passes through the center of the elliptical galaxy. Equations (26) and (32) can be inverted numerically to find the radius and velocity as a function of time, $r=r(\Delta t)$ and $v=v(\Delta t)$, respectively.

In Fig. 4, we plot $\mathbf{r} / r_{0}, \mathbf{v} / \nu_{0}$, and $(\mathbf{a} / 100 \tilde{a})$ versus the scaled time $t / T$. We plot $\mathbf{a} / 100 \tilde{a}$ to better capture the divergence of the acceleration as $r \rightarrow 0$. As for $\gamma=1$, the timedependent radius is approximately a cosine function, $\mathbf{r}(t)$ $\simeq r_{0} \cos (2 \pi t / T)$. The time-dependent velocity and acceleration do not correspond to harmonic motion. We find that the velocity $\mathbf{v}(t)$ is a concave function for $0<t<T / 4$, whereas the velocity for harmonic motion is convex for the same time

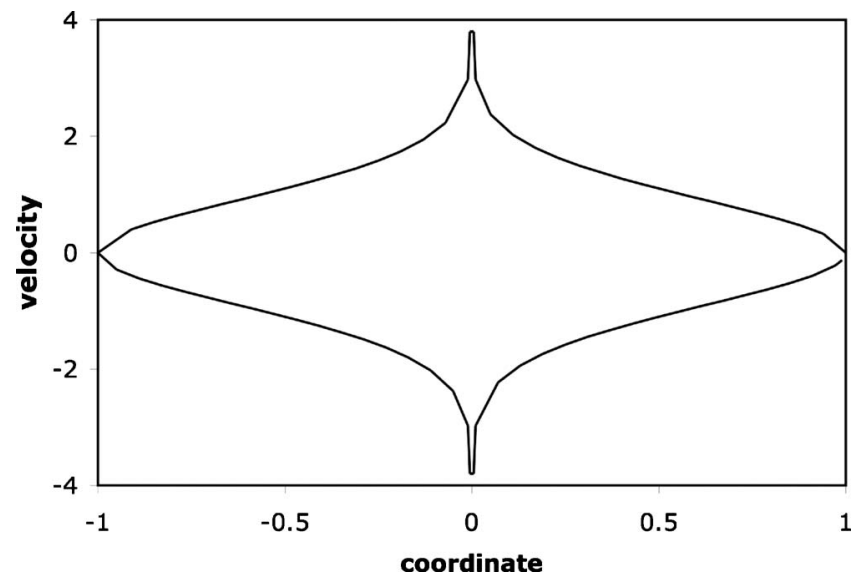

Fig. 5. The phase portrait for the strong-cusp limit.

interval. The star's acceleration is markedly faster than that for harmonic motion, and the acceleration is very small except very close to the center of the elliptical galaxy.

We plot the phase portrait of the motion in Fig. 5. In the upper half, $v>0$, the phase portrait is concave only near the turning points, $\mathbf{r}= \pm r_{0}$. Because the acceleration diverges as $r \rightarrow 0$, the plot is strongly convex near the center. The convex behavior for $\gamma=2$ is the continuation of the cusplike behavior for $\gamma=1$.

The solution of the equation of motion for $\gamma=3 / 2$ cannot be expressed in terms of elementary functions. We used the Runge-Kutta differential equation solvers in MATHCAD. As expected, the behavior for $\gamma=3 / 2$ interpolates between that for $\gamma=1$ and $\gamma=2$. We find $v_{\max } \simeq 3.26 \widetilde{v}$ for $r_{0} / R_{1}=0.2$. We plot the radius, velocity, and acceleration in Fig. 6 as a function of time; the phase portrait is shown in Fig. 7. The curve is not closed due to our use of the fourth-order Runge-Kutta algorithm. This inaccuracy could be addressed by using an adaptive step size algorithm or working with the inverse radius $r^{-1}$.

\section{DISCUSSION}

We have discussed radial motion in a central potential produced by a singular mass distribution such that $M(r) \sim r^{\alpha}$ for the mass inside the radius $r$. For $0<\alpha<3$, this mass density

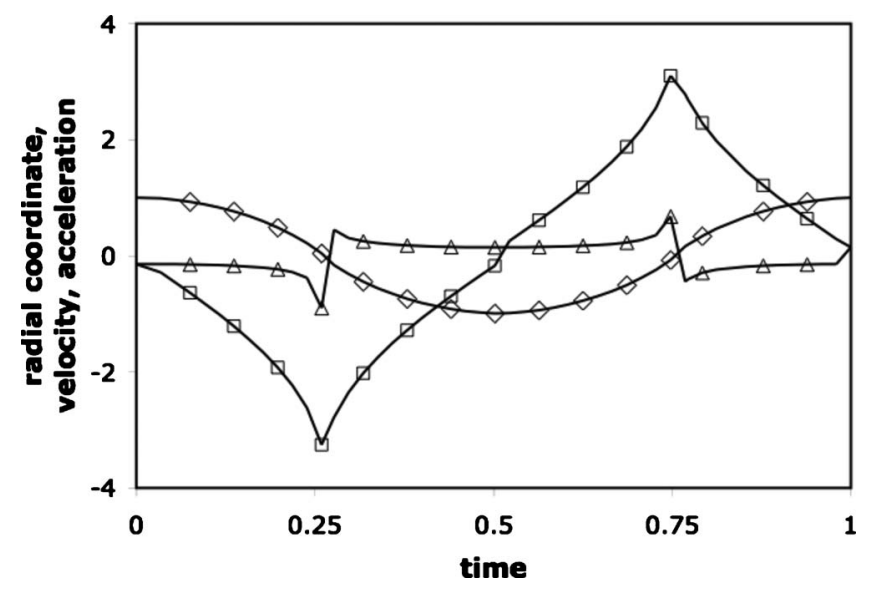

Fig. 6. The scaled radial coordinate $(\triangle)$, velocity $(\square)$, and acceleration $(\triangle)$ in the limit $\gamma=1.5$. 


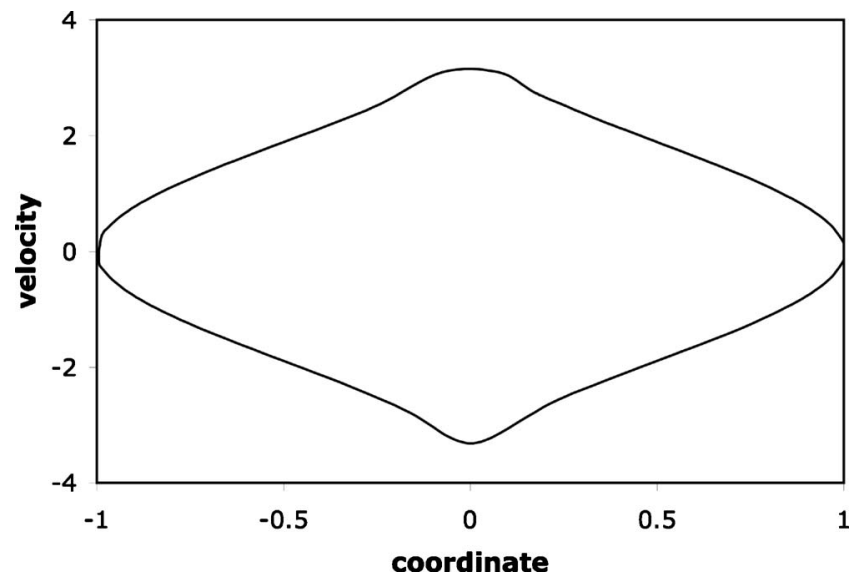

Fig. 7. The phase portrait for $\gamma=1.5$.

extrapolates between the two cases for which bound orbits are closed, the Kepler problem for $\alpha=0$ and the isotropic oscillator for $\alpha=3$.

Because the acceleration is nonzero at $r=0$, the phase portrait does not have the elliptical shape found for harmonic motion. The speed is finite in the weak-cusp limit, $\gamma=1$, and diverges in the strong-cusp limit, $\gamma=2$. We found closedform expressions for $r(t)$ for $\gamma=1$. For $\gamma=2$, closed-form expressions were found only in the limit as the star is passing through the center of galaxy. The divergence of the velocity occurs for a very short time interval compared to the period of motion.

The dependence of the period on the amplitude equal to the initial radius $r_{0}$ is given for radial motion by

$$
\frac{T}{\tilde{T}} \sim\left(\frac{r_{0}}{R_{1}}\right)^{\gamma / 2} \quad(\gamma=1,2) .
$$

For $r_{0} / R_{1}=0.2$, we find $T / \tilde{T} \simeq 2.53$ and $T / \tilde{T} \simeq 1.00$ for $\gamma=1$ and $\gamma=2$, respectively. For comparison, we also plot the period for harmonic motion, $T / \widetilde{T}=2 \pi$. We find that a singular mass distribution shortens the periodicity of radial motion. This behavior is shown in Fig. 8. The period for orbital motion $T_{\text {orb }}$ is calculated from $\left(2 \pi / T_{\text {orb }}\right)^{2} r=a(r)$ and we find

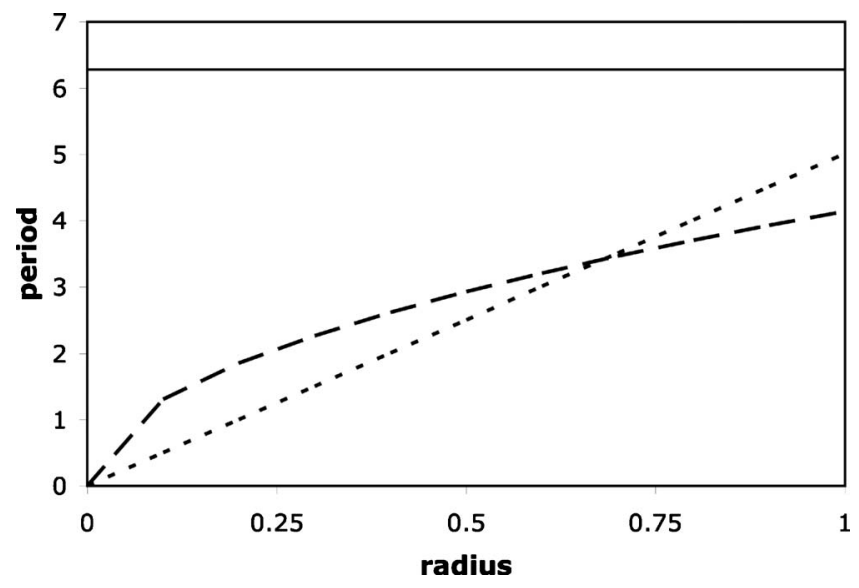

Fig. 8. The radial dependence of the period for the harmonic potential (solid line), weak-cusp limit (long dash line), and strong-cusp limit (short dash line).

$$
\frac{T_{\text {orb }}}{\widetilde{T}}=2 \pi\left(\frac{r_{0}}{R_{1}}\right)^{\gamma / 2}\left(1+\frac{r_{0}}{R_{1}}\right)^{1 / \gamma} \quad(\gamma=1,2) .
$$

We thus find that for arbitrary values of $\gamma$, the periods for radial and orbital motion are incommensurate, $T / T_{\text {orb }}$ $\neq n_{1} / n_{2}$ for (positive) integers $n_{1}$ and $n_{2}$ for arbitrary values of the initial radius $r_{0} / R_{1}$. This behavior suggests that bound orbits of stars inside elliptical galaxies do not close on themselves.

A star moves along the radius of a galaxy if the galaxy's mass distribution is isotropic. Our calculations are relevant to this situation. We discussed the trajectories of stars for a given galaxy mass distribution. The structure and dynamics of galaxies are related to each other, and the trajectories of all the stars in the galaxy determine its mass distribution. It was discovered in the mid-1970s that elliptical galaxies exhibit a rich variety of shapes and internal dynamics. ${ }^{17}$ For this case, Schwarzschild ${ }^{18}$ showed that self-consistent mass densities of galaxies can be obtained from the orbital dynamics of stars. Similar conclusions were reached by Vandervoort based on many-body simulation studies. ${ }^{19}$

\section{ACKNOWLEDGMENTS}

The authors would like to acknowledge constructive and helpful comments by two anonymous referees.

${ }^{a)}$ Author to whom correspondence should be addressed. Electronic mail: u.zurcher@csuohio.edu

${ }^{1}$ S. T. Thornton and J. B. Marion, Classical Dynamics of Particles and Systems, 5th ed. (Thomson-Brooks/Cole, Belmont, CA, 2004), Chap. 8.

${ }^{2}$ A. P. French, Newtonian Mechanics (Norton, New York, 1971), p. 452.

${ }^{3}$ L. Carroll, Alice in Wonderland and Through the Looking Glass (Oxford U. P., New York, 2009).

${ }^{4}$ L. Frank Baum, Dorothy and the Wizard in $\mathrm{Oz}$ (Harper Collins, New York, 1990).

${ }^{5}$ The Annotated Alice: The Definitive Edition, edited by M. Gardner (W. W. Norton, New York, 1999).

${ }^{6}$ Galileo Galilei, Dialogue Concerning the Two Chief World SystemsPtolemaic and Copernican. Translated by S. Drake (U. of California Press, Los Angeles, CA, 1953).

${ }^{7}$ D. Merritt, "Chaos and the shape of elliptical galaxies," Science 271, 337-340 (1996).

${ }^{8}$ H. Shapley, "Galactic and extragalactic studies: XIII. Note on the comparative diameters of spheroidal and spiral galaxies," Proc. Natl. Acad. Sci. U.S.A. 28 (5), 186-191 (1942).

${ }^{9}$ G. de Vaucouleurs, "Classification and morphology of external galaxies," Handbuch der Physik (Springer-Verlag, Berlin, 1959), Vol. 53, pp. 275311.

${ }^{10}$ L. S. Sparke and J. S. Gallagher III, Galaxies in the Universe, 2 nd ed. (Cambridge U. P., Cambridge, 2004).

${ }^{11}$ W. Dehnen, "A family of potential densities pairs for spherical galaxies and bulges," Mon. Not. R. Astron. Soc. 265, 250-256 (1993).

${ }^{12}$ L. Hernquist, "An analytical model for spherical galaxies and bulges," Astrophys. J. 356, 359-364 (1990).

${ }^{13}$ W. Jaffe, "A simple model for the distribution of light in spherical galaxies," Mon. Not. R. Astron. Soc. 202, 995-999 (1983).

${ }^{14}$ MATHCAD, PTC Inc., Needham, MA 02494.

${ }^{15}$ C. W. Misner, K. S. Thorne, and J. A. Wheeler, Gravitation (Freeman, San Francisco, 1970). 
${ }^{16}$ M. Abramowitz and I. A. Stegun, Handbook of Mathematical Functions (Dover, New York, 1965), Formula 7.1.23.

${ }^{17}$ T. de Zeeuw and M. Franx, "Structure and dynamics of elliptical galaxies," Annu. Rev. Astron. Astrophys. 29, 239-274 (1991).
${ }^{18}$ M. Schwarzschild, "Triaxial equilibrium-models for elliptical galaxies with slow figure rotation," Astrophys. J. 263, 599-610 (1982).

${ }^{19}$ P. O. Vandervoort, "On Lagrangian methods for the study of small perturbations in elliptical galaxies," Astrophys. J. 341, 105-112 (1989).

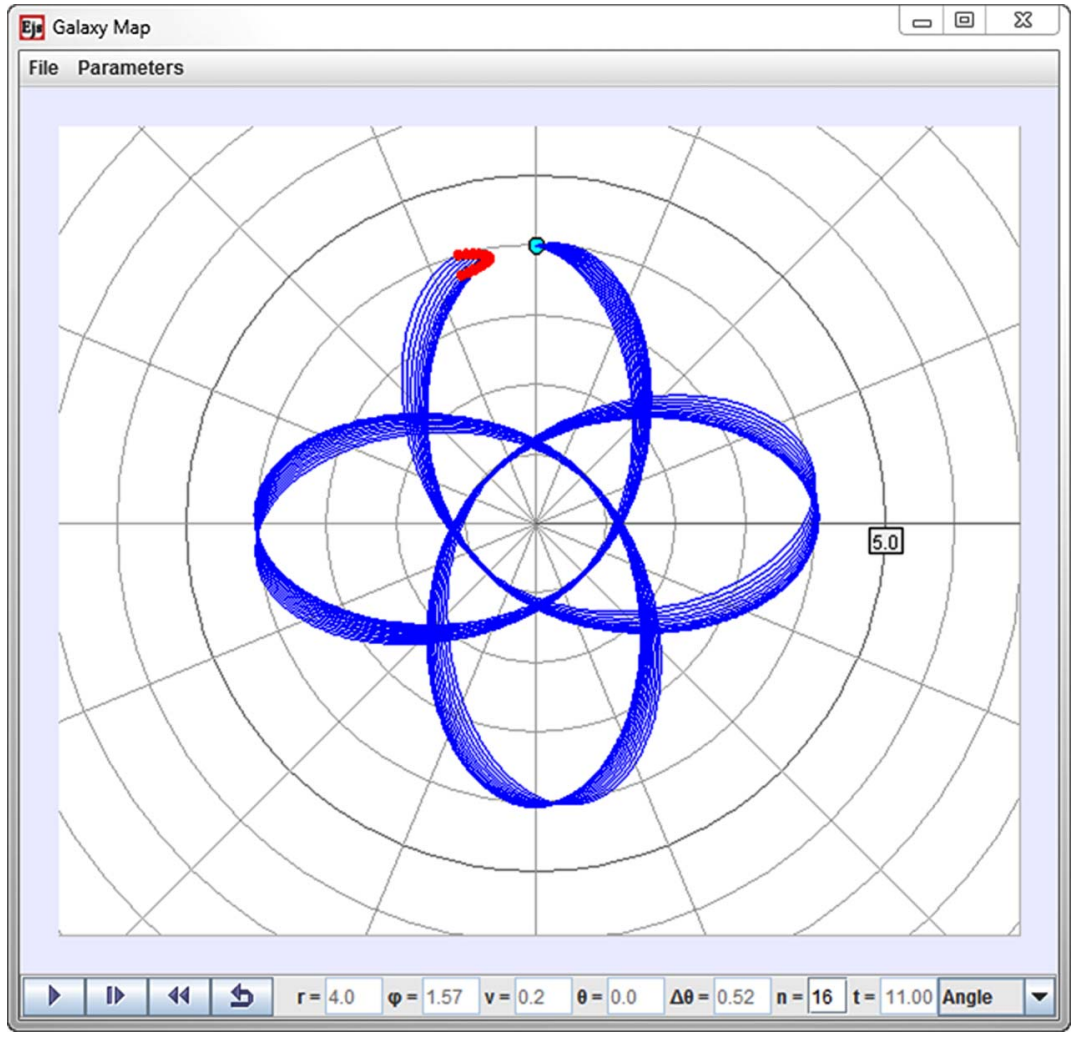

The Orbits Within Spherical Galaxies model displays the two-dimensional trajectories of particles (stars) within a galaxy having a spherically symmetric mass distribution that heuristically approximates the distributions found in galaxies and bulges. The model uses a mass density proposed by Walter Dehnen in "A family of potential-density pairs for spherical galaxies and bulges" [Mon. Not. R. Astron. Soc. 265, 250-256 (1993)] to describe spatial distributions that vary as $r^{-4}$ and $r^{-\gamma}$ in galactic envelopes and cores where $\gamma$ is an adjustable power-law parameter. The simulation's main window lets users vary the number of particles and their initial conditions using input fields and a drop-down menu. A second window shows that the Dehnen model potential function and a $1 / r^{2}$ function for comparison. Units are chosen such that a typical galaxy has total mass $M=1$ and the gravitational constant $G=1$.

http://www.compadre.org/OSP/items/detail.cfm?ID=10583

The Orbits Within Spherical Galaxies model is a supplemental simulation for the paper by Ulrich Zürcher and Miron Kaufman and it has been approved by the authors and the AJP editor. Partial funding for the development of these models was obtained through NSF Grant No. DUE-0937731. 